\title{
UNIQUENESS AND LOCAL EXISTENCE OF SOLUTIONS TO AN APPROXIMATE SYSTEM OF A 1D SIMPLIFIED TUMOR INVASION MODEL
}

\author{
MACIEJ CYTOWSKI \\ Interdisciplinary Centre for Mathematical and Computational Modelling \\ Warsaw University \\ Pawińskiego 5a, 02-106 Warszawa, Poland \\ E-mail: M.Cytowski@icm.edu.pl \\ AKIO ITO \\ Department of Electronic Engineering and Computer Science \\ School of Engineering, Kinki University \\ 1 Takayaumenobe, Higashihiroshimashi, Hiroshima, 739-2116, Japan \\ E-mail: aito@hiro.kindai.ac.jp \\ MAREK NIEZGÓDKA \\ Interdisciplinary Centre for Mathematical and Computational Modelling \\ Warsaw University \\ Pawińskiego 5a, 02-106 Warszawa, Poland \\ E-mail:marekn@icm.edu.pl
}

\begin{abstract}
In the present paper, we consider an approximate system of one-dimensional simplified tumor invasion model, which was originally proposed by Chaplain and Anderson in [1]. The simplified tumor invasion model is composed of PDE and ODE. Actually, the PDE is the balance equation of the density of tumor cells and the ODE describes the dynamics of concentration of extracellular matrix. In this model, we take into account that the random motility of the density of tumor cells is given by a function of space and time, that is, it is not a positive constant. Moreover, the PDE contains a (nonlinear) function which describes the proliferation as well as the apoptosis of tumor cells. Our main objective is to give the local existence and uniqueness of the solutions to the approximate system.
\end{abstract}

2000 Mathematics Subject Classification: 35Q80, 35M99.

Key words and phrases: tumor invasion model, uniqueness and existence of solutions. The paper is in final form and no version of it will be published elsewhere. 
1. Introduction. We propose the following one-dimensional tumor invasion model denoted by $(\mathrm{P}):=\{(1)-(5)\}$ throughout this paper:

$$
\begin{gathered}
n_{t}=\left[p(x, t) n_{x}-n(\varphi(v))_{x}\right]_{x}+F(x, t, n, v) \quad \text { a.e. in } Q_{T}:=(-L, L) \times(0, T), \\
v_{t}=\nu n \quad \text { a.e. in } Q_{T}, \\
{\left[p n_{x}-n(\varphi(v))_{x}\right]( \pm L, t)=0 \quad \text { a.e. } t \in(0, T),} \\
n(x, 0)=n_{0}(x) \quad \text { a.e. } x \in(-L, L), \\
v(x, 0)=v_{0}(x) \quad \text { a.e. } x \in(-L, L),
\end{gathered}
$$

where $p$ is a non-negative and bounded function from $(-L, L) \times(0, T) ; \varphi$ is a function from $\mathbf{R}$ into itself; $F$ is a function from $(-L, L) \times(0, T) \times \mathbf{R} \times \mathbf{R} ; T, L$ and $\nu$ are given positive and finite numbers; $n_{0}$ and $v_{0}$ are the prescribed initial data.

Phenomenologically, $n$ and $v$ reflect the density of tumor cells and some quantity concerned with the concentration of extracellular matrix, respectively.

This system comes from the following one-dimensional tumor invasion models, which is one of the simplified models proposed by Chaplain and Anderson in [1]:

$$
\begin{gathered}
n_{t}=\left[D_{n} n_{x}-n \chi(f) f_{x}\right]_{x} \text { a.e. in } Q_{T}, \\
f_{t}=-\nu m f \text { a.e. in } Q_{T}, \\
m_{t}=D_{m} m_{x x}+g(n, m)-h(n, m, f)-k(m, u) \text { a.e. in } Q_{T}, \\
u_{t}=D_{u} m_{x x}+\ell(m, f)-k(m, u)-\varepsilon u \quad \text { a.e. in } Q_{T},
\end{gathered}
$$

where $f, m$ and $u$ are the densities of extracellular matrix, enzyme denatured extracellular matrix and endogenous inhibitor, respectively. $D_{n}, D_{m}, D_{u}$ and $\varepsilon$ are positive constants and $g, h, k$ and $\ell$ are non-negative functions.

Now, we do not consider the existence of endogenous inhibitor, namely, $u \equiv 0$. Moreover, we assume that tumor cells directly denature extracellular matrix. Roughly speaking, we identify the behavior of enzyme denatured extracellular matrix as that of tumor cells. Then, we derive the following simplified tumor invasion model denoted by $(\mathrm{SP})=\{(10),(11)\}$ :

$$
\begin{gathered}
n_{t}=\left[D_{n} n_{x}-n \chi(f) f_{x}\right]_{x} \quad \text { a.e. in } Q_{T}, \\
f_{t}=-\nu n f \quad \text { a.e. in } Q_{T},
\end{gathered}
$$

There are several papers, for example $[2,3,4,5]$, in which the authors treated (SP).

In the present paper, we propose the following one-dimensional tumor invasion model of Chaplain-Anderson type:

$$
n_{t}=\left[p(x, t) n_{x}-\chi(f) n f_{x}\right]_{x}+\bar{F}(x, t, n, f)
$$

with (11). The differences between (6) and (12) are the following two facts:

1. The random motility of $n$, denoted by $p$ in (12), is given by a function of space and time in (12), but in (6) it is a positive constant $D_{n}$. 
2. (12) contains a (nonlinear) function $\bar{F}$, which describes the proliferation and the apoptosis of tumor cells.

By putting $v=-\log f, \varphi(r)=\hat{\chi}\left(e^{-r}\right)(\forall r \in \mathbf{R})$, where $\hat{\chi}$ is a primitive of $\chi$, and $F(x, t, n, v)=\bar{F}\left(x, t, n, e^{-v}\right)$, we can easily derive (1) and (2) from (12) and (7), respectively. Namely, we can consider $\{(7),(12)\}$ as one of the special cases of the form $\{(1),(2)\}$.

Next, we give the notation and the mathematical assumptions on the prescribed data.

We denote by $H:=L^{2}(-L, L)$ a Hilbert space with the usual $L^{2}$-inner product $(\cdot, \cdot)_{H}$ and the norm $\|\cdot\|_{H}$, and by $V:=H^{1}(-L, L)$ a Hilbert space with the inner product $(\cdot, \cdot)_{V}$ given by

$$
\left(z_{1}, z_{2}\right)_{V}=\left(z_{1}, z_{2}\right)_{H}+\kappa\left(\left(z_{1}\right)_{x},\left(z_{2}\right)_{x}\right)_{H}, \quad \forall z_{1}, z_{2} \in V .
$$

Moreover, we denote by $\|\cdot\|_{V}, V^{*}$ and $\langle\cdot, \cdot\rangle_{V^{*}, V}$ the norm of $V$ induced by $(\cdot, \cdot)_{V}$, the dual space of $V$ and the duality pairing between $V^{*}$ and $V$, respectively.

We suppose that the functions $p, \varphi, F$ and the initial data $n_{0}, v_{0}$ satisfy the following conditions:

(A1) $p$ is a bounded function from $(-L, L) \times(0, T)$ into $[0,+\infty)$, that is, there exists a positive constant $c_{1}$ such that

$$
0 \leq p(x, t) \leq c_{1}, \quad \text { a.e. }(x, t) \in(-L, L) \times(0, T) .
$$

(A2) $\varphi$ is a Lipschitz continuous $C^{1}$ function from $\mathbf{R}$ into itself whose Lipschitz constant is denoted by $L(\varphi)$. Moreover, we assume that the derivative of $\varphi$, denoted by $\varphi^{\prime}$, is also a Lipschitz continuous function whose Lipschitz constant is $L\left(\varphi^{\prime}\right)$.

(A3) $F$ is a continuous function from $[-L, L] \times[0, T] \times \mathbf{R} \times \mathbf{R}$ into $\mathbf{R}$. Moreover, we assume that

$$
F(x, t, 0, r)=0, \quad \forall(x, t, r) \in[-L, L] \times[0, T] \times \mathbf{R}
$$

and there exists a positive constant $c_{2}$ such that

$$
\begin{gathered}
\left|F\left(x, t, r_{1}, s_{1}\right)-F\left(x, t, r_{2}, s_{2}\right)\right| \leq c_{2}\left(\left|r_{1}-r_{2}\right|+\left|s_{1}-s_{2}\right|\right), \\
\forall x \in[-L, L], \forall t \in[0, T], \forall r_{i}, s_{i} \in \mathbf{R}(i=1,2) .
\end{gathered}
$$

(A4) $n_{0} \in V$.

(A5) $v_{0} \in V$.

From (A1), it is difficult to show the existence and uniqueness of solutions to (P). So, in the present paper, for each $\kappa>0$ we consider the following approximate system of $(\mathrm{P})$, denoted by $(\mathrm{P})_{\kappa}:=\{(13)-(17)\}$ :

$$
\begin{gathered}
n_{t}=\left[\kappa n_{t x}+p(x, t) n_{x}-n(\varphi(v))_{x}\right]_{x}+F(x, t, n, v) \quad \text { a.e. in } Q_{T}, \\
v_{t}=\nu n \quad \text { a.e. in } Q_{T}, \\
{\left[\kappa n_{t x}+p n_{x}-n(\varphi(v))_{x}\right]( \pm L, t)=0 \quad \text { a.e. } t \in(0, T),} \\
n(x, 0)=n_{0}(x) \quad \text { a.e. } x \in(-L, L), \\
v(x, 0)=v_{0}(x) \quad \text { a.e. } x \in(-L, L),
\end{gathered}
$$


By adding the term $\kappa n_{t x}$, we make the solution $[n, v]$ regularized in a suitable function space (cf. Definition 1.1 below).

Definition 1.1. A pair $[n, v]$ of functions $n:[0, T] \rightarrow V$ and $v:[0, T] \rightarrow V$ is called a solution to $(\mathrm{P})$ on $[0, T]$ iff the following conditions (i)-(iv) are fulfilled:

(i) $[n, v] \in W^{1, \infty}(0, T ; V) \times W^{2, \infty}(0, T ; V)$.

(ii) For any $z \in V$ and a.e. $t \in(0, T)$ the following equality holds:

$$
\left(n_{t}(t), z\right)_{V}+\int_{-L}^{L} p(x, t) n_{x}(t) z_{x} d x-\int_{-L}^{L} n(t)(\varphi(v))_{x}(t) z_{x} d x=\int_{-L}^{L} F(x, t, n(t), v(t)) z d x .
$$

(iii) (2) is satisfied.

(iv) $n(0)=n_{0}$ and $v(0)=v_{0}$ in $V$.

2. Uniqueness of solutions. We devote this section to showing the uniqueness of solutions to $(\mathrm{P})$ on $[0, T]$. Since the space dimension is 1 , we note that the following imbedding holds: there exists a positive constnat $c_{3}$ such that

$$
\|z\|_{C[-L, L]} \leq c_{3}\|z\|_{V}, \quad \forall z \in V,
$$

which plays a crucial role throughout this paper.

The main theorem in this section is given below.

Theorem 2.1. Assume that (A1)-(A5) hold. Then, (P) has at most one solution $[n, v]$ on $[0, T]$.

Proof. Let $\left[n_{i}, v_{i}\right](i=1,2)$ be two solutions to $(\mathrm{P})$ on $[0, T]$ and put $N=n_{1}-n_{2}$. Then, $N$ satisfies the following equality:

$$
\begin{gathered}
\left(N_{t}(t), z\right)_{V}+\int_{-L}^{L} p(x, t) N_{x}(t) z_{x} d x-\int_{-L}^{L} n_{1}(t) \varphi^{\prime}\left(v_{1}(t)\right)\left(v_{1}\right)_{x}(t) z_{x} d x \\
+\int_{-L}^{L} n_{2}(t) \varphi^{\prime}\left(v_{2}(t)\right)\left(v_{2}\right)_{x}(t) z_{x} d x \\
=\int_{-L}^{L}\left\{F\left(x, t, n_{1}(t), v_{1}(t)\right)-F\left(x, t, n_{2}(t), v_{2}(t)\right)\right\} z d x, \\
\forall z \in V \text {, a.e. } t \in(0, T) .
\end{gathered}
$$

We substitute $z=N(t)$ in (19) and use (A1) to derive

$$
\frac{1}{2} \frac{d}{d t}\|N(t)\|_{V}^{2} \leq \sum_{i=1}^{4} I_{i}(t), \quad \text { a.e. } t \in(0, T)
$$

where

$$
\begin{aligned}
& I_{1}(t)=\int_{-L}^{L}\left|F\left(x, t, n_{1}(t), v_{1}(t)\right)-F\left(x, t, n_{2}(t), v_{2}(t)\right)\right||N(t)| d x, \\
& I_{2}(t)=\int_{-L}^{L}\left|\varphi^{\prime}\left(v_{1}(t)\right)\left\|\left(v_{1}\right)_{x}(t)\right\|\right| N(t)|| N_{x}(t) \mid d x,
\end{aligned}
$$




$$
\begin{aligned}
& I_{3}(t)=\int_{-L}^{L}\left|n_{2}(t)\left\|\varphi^{\prime}\left(v_{1}(t)\right)-\varphi^{\prime}\left(v_{2}(t)\right)\right\|\left(v_{1}\right)_{x}(t) \| N_{x}(t)\right| d x, \\
& I_{4}(t)=\int_{-L}^{L}\left|n_{2}(t)\left\|\varphi^{\prime}\left(v_{2}(t)\right)\right\|\left(v_{1}\right)_{x}(t)-\left(v_{2}\right)_{x}(t) \| N_{x}(t)\right| d x .
\end{aligned}
$$

We estimate the integrals $I_{i}(1 \leq i \leq 4)$ in the following way:

(i) It follows from (A3), (2) and (18) that

$$
\begin{aligned}
I_{1}(t) & \leq c_{2} \int_{-L}^{L}\left(|N(t)|+\left|v_{1}(t)-v_{2}(t)\right|\right)|N(t)| d x \\
& \leq c_{2}\|N(t)\|_{H}^{2}+\nu c_{2} \int_{-L}^{L}\left(\int_{0}^{t}|N(s)| d s\right)|N(t)| d x \\
& \leq c_{2}\|N(t)\|_{V}^{2}+\nu c_{2}\left(\int_{0}^{t}\|N(s)\|_{V} d s\right)\|N(t)\|_{V} .
\end{aligned}
$$

(ii) It follows from (A2) with (2) and (18) again that

- $I_{2}(t) \leq c_{3} L(\varphi)\|N(t)\|_{V}\left\|\left(v_{1}\right)_{x}(t)\right\|_{H}\left\|N_{x}(t)\right\|_{H}$

$$
\begin{aligned}
& \leq \frac{c_{3} L(\varphi)}{\kappa}\left\|v_{1}(t)\right\|_{V}\|N(t)\|_{V}^{2} \\
& \leq \frac{c_{3} L(\varphi)\left\|v_{1}\right\|_{L^{\infty}(0, T ; V)}}{\kappa}\|N(t)\|_{V}^{2}
\end{aligned}
$$

- $I_{3}(t) \leq \nu L\left(\varphi^{\prime}\right) \int_{-L}^{L}\left|n_{2}(t)\right|\left(\int_{0}^{t}|N(s)| d s\right)\left|\left(v_{1}\right)_{x}(t)\right|\left|N_{x}(t)\right| d x$

$$
\begin{aligned}
& \leq \nu c_{3}^{2} L\left(\varphi^{\prime}\right)\left\|n_{2}(t)\right\|_{V}\left(\int_{0}^{t}\|N(s)\|_{V} d s\right)\left\|\left(v_{1}\right)_{x}(t)\right\|_{H}\left\|N_{x}(t)\right\|_{H} \\
& \leq \frac{\nu c_{3}^{2} L\left(\varphi^{\prime}\right)\left\|n_{2}(t)\right\|_{V}\left\|v_{1}(t)\right\|_{V}}{\kappa}\left(\int_{0}^{t}\|N(s)\|_{V} d s\right)\|N(t)\|_{V} \\
& \leq \frac{\nu c_{3}^{2} L\left(\varphi^{\prime}\right)\left\|n_{2}\right\|_{L^{\infty}(0, T ; V)}\left\|v_{1}\right\|_{L^{\infty}(0, T ; V)}}{\kappa}\left(\int_{0}^{t}\|N(s)\|_{V} d s\right)\|N(t)\|_{V} ;
\end{aligned}
$$

- $I_{4}(t) \leq \nu L(\varphi) \int_{-L}^{L}\left|n_{2}(t)\right|\left(\int_{0}^{t}\left|N_{x}(s)\right| d s\right)\left|N_{x}(t)\right| d x$

$$
\begin{aligned}
& \leq \nu c_{3} L(\varphi)\left\|n_{2}(t)\right\|_{V}\left(\int_{0}^{t}\left\|N_{x}(s)\right\|_{H} d s\right)\left\|N_{x}(t)\right\|_{H} \\
& \leq \frac{\nu c_{3} L(\varphi)\left\|n_{2}(t)\right\|_{V}}{\kappa}\left(\int_{0}^{t}\|N(s)\|_{V} d s\right)\|N(t)\|_{V} \\
& \leq \frac{\nu c_{3} L(\varphi)\left\|n_{2}\right\|_{L^{\infty}(0, T ; V)}}{\kappa}\left(\int_{0}^{t}\|N(s)\|_{V} d s\right)\|N(t)\|_{V} .
\end{aligned}
$$


By substituting the estimates obtained in (i) and (ii) into (20), we have:

$$
\frac{d}{d t}\|N(t)\|_{V}^{2} \leq C_{1}\|N(t)\|_{V}^{2}+C_{2} \frac{d}{d t}\left(\int_{0}^{t}\|N(s)\|_{V} d s\right)^{2}, \quad \text { a.e } t \in(0, T),
$$

where

$$
C_{1}=2 c_{2}+\frac{2 c_{3} L(\varphi)\left\|v_{1}\right\|_{L^{\infty}(0, T ; V)}}{\kappa}
$$

and

$$
C_{2}=2 \nu c_{2}+\frac{2 \nu c_{3}^{2} L\left(\varphi^{\prime}\right)\left\|n_{2}\right\|_{L^{\infty}(0, T ; V)}\left\|v_{1}\right\|_{L^{\infty}(0, T ; V)}}{\kappa}+\frac{2 \nu c_{3} L(\varphi)\left\|n_{2}\right\|_{L^{\infty}(0, T ; V)}}{\kappa} .
$$

Now, we integrate $(21)$ on $[0, t](\subset[0, T])$ and obtain

$$
\|N(t)\|_{V}^{2} \leq C_{1} \int_{0}^{t}\|N(s)\|_{V}^{2} d s+C_{2}\left(\int_{0}^{t}\|N(s)\|_{V} d s\right)^{2}, \quad \forall t \in[0, T],
$$

that is,

$$
\|N(t)\|_{V}^{2} \leq\left(C_{1}+C_{2} T\right) \int_{0}^{t}\|N(s)\|_{V}^{2} d s, \quad \forall t \in[0, T] .
$$

At last, by applying the Gronwall lemma to (22), we have $n_{1} \equiv n_{2}$ in $V$ on $[0, T]$, so, $v_{1} \equiv v_{2}$ in $V$ on $[0, T]$. This implies the uniqueness of solutions to $(\mathrm{P})$ on $[0, T]$.

3. Auxiliary problem. Throughout this section, we fix any function $\ell \in W^{1, \infty}(0, T ; V)$ with $\ell(0)=n_{0}$. And we see from (A5) that the following lemma holds.

Lemma 3.1. We put

$$
\eta_{\ell}(x, t)=v_{0}(x)+\nu \int_{0}^{t} \ell(x, s) d s, \quad \forall(x, t) \in[-L, L] \times[0, T] .
$$

Then, we have $\eta_{\ell} \in W^{2, \infty}(0, T ; V)$.

Now, we consider the following auxiliary problem $(\mathrm{AP})_{\ell}$ : find a function $w \in$ $W^{1, \infty}(0, T ; V)$ satisfying

$$
\begin{aligned}
\left(w_{t}(t), z\right)_{V} & +\int_{-L}^{L} p(x, t) w_{x}(t) z_{x} d x-\int_{-L}^{L}\left(\varphi\left(v_{0}\right)\right)_{x} w(t) z_{x} d x \\
& =\int_{-L}^{L} F\left(x, t, n_{0}, v_{0}\right) z d x+\left\langle F_{\ell}^{*}(t), z\right\rangle_{V^{*}, V}, \quad \forall z \in V, \quad \text { a.e. } t \in(0, T)
\end{aligned}
$$

with the initial condition:

$$
w(x, 0)=n_{0}(x) \quad \text { a.e. } x \in(-L, L),
$$

where for each $t \in[0, T] F_{\ell}^{*}(t) \in V^{*}$ is defined by

$$
\begin{aligned}
\left\langle F_{\ell}^{*}(t), z\right\rangle_{V^{*}, V}=\int_{-L}^{L}\{F(x, t, \ell(t) & \left.\left., \eta_{\ell}(t)\right)-F\left(x, t, n_{0}, v_{0}\right)\right\} z d x \\
& +\int_{-L}^{L} \ell(t)\left(\varphi\left(\eta_{\ell}(t)\right)-\varphi\left(v_{0}\right)\right)_{x} z_{x} d x
\end{aligned}
$$

Then, we have the following proposition. 
Proposition 3.1. (AP) $)_{\ell}$ has a unique solution $w \in W^{1, \infty}(0, T ; V)$. Moreover, there exists a strictly increasing and locally bounded function $h_{1}:[0,+\infty) \rightarrow(3,+\infty)$ such that

$$
\|w\|_{W^{1, \infty}(0, t ; V)} \leq h_{1}(t)\left(\left\|n_{0}\right\|_{V}+\left\|v_{0}\right\|_{V}+\left\|F_{\ell}^{*}\right\|_{L^{\infty}\left(0, t ; V^{*}\right)}\right), \quad \forall t \in[0, T] .
$$

Proof. By using the Galerkin method, we can easily show that $(\mathrm{AP})_{\ell}$ has a solution $w$ and we omit it in this proof because it is a quite standard argument.

In order to show the uniqueness of the solutions to $(\mathrm{AP})_{\ell}$, let $w_{i}(i=1,2)$ be two solutions to $(\mathrm{AP})_{\ell}$ on $[0, T]$. Then, we see that $W=w_{1}-w_{2}$ satisfies

$$
\begin{gathered}
\left(W_{t}(t), z\right)_{V}+\int_{-L}^{L} p(x, t) W_{x}(t) z_{x} d x-\int_{-L}^{L}\left(\varphi\left(v_{0}\right)\right)_{x} W(t) z_{x} d x=0, \\
\forall z \in V, \quad \text { a.e. } t \in(0, T),
\end{gathered}
$$

with the initial condition:

$$
W(x, 0)=0 \quad \text { a.e. } x \in(-L, L) .
$$

We substitute $z=W(t)$ in (26) and use (A1) with (18) to obtain

$$
\frac{d}{d t}\|W(t)\|_{V}^{2} \leq \frac{2 c_{3} L(\varphi)\left\|v_{0}\right\|_{V}}{\kappa}\|W(t)\|_{V}^{2}, \quad \text { a.e. } t \in(0, T) .
$$

By applying the Gronwall lemma to the above inequality with (27), we see $w_{1} \equiv w_{2}$ in $V$ on $[0, T]$.

In the rest of this proof, we show the existence of a function $h_{1}$ satisfying (25). For this, we substitute $z=w(t)$ in (23) to obtain

$$
\begin{gathered}
\frac{1}{2} \frac{d}{d t}\|w(t)\|_{V}^{2}+\int_{-L}^{L} p(x, t)\left|w_{x}(t)\right|^{2} d x-\int_{-L}^{L} \varphi^{\prime}\left(v_{0}\right)\left(v_{0}\right)_{x} w(t) w_{x}(t) d x \\
=\int_{-L}^{L} F\left(x, t, n_{0}, v_{0}\right) w(t) d x+\left\langle F_{\ell}^{*}(t), w(t)\right\rangle_{V^{*}, V}, \quad \text { a.e. } t \in(0, T) .
\end{gathered}
$$

We see from (A1)-(A5) that the following estimates hold:

$$
\begin{aligned}
& -\int_{-L}^{L} p(x, t)\left|w_{x}(t)\right|^{2} d x \geq 0 ; \\
& \text { - } \int_{-L}^{L}\left|\varphi^{\prime}\left(v_{0}\right)\left\|\left(v_{0}\right)_{x}|| w(t)\right\| w_{x}(t)\right| d x \leq \frac{c_{3} L(\varphi)\left\|v_{0}\right\|_{V}}{\kappa}\|w(t)\|_{V}^{2} ; \\
& \text { - } \int_{-L}^{L}\left|F\left(x, t, n_{0}, v_{0}\right)\left\|w(t) \mid d x \leq c_{2}\left(\left\|n_{0}\right\|_{V}+\left\|v_{0}\right\|_{V}\right)\right\| w(t) \|_{V} .\right.
\end{aligned}
$$

By using these estimates, we see that there exist constants $C_{i}>0(i=3,4)$ such that

$$
\frac{d}{d t}\|w(t)\|_{V}^{2} \leq C_{3}\|w(t)\|_{V}^{2}+C_{4}\left(\left\|n_{0}\right\|_{V}^{2}+\left\|v_{0}\right\|_{V}^{2}+\left\|F_{\ell}^{*}(t)\right\|_{V^{*}}^{2}\right), \quad \text { a.e. } t \in(0, T) .
$$

By applying the Gronwall lemma to the above inequality, we have:

$$
\|w\|_{L^{\infty}(0, t ; V)} \leq \sqrt{\left(1+C_{4} t\right) e^{C_{3} t}}\left(\left\|n_{0}\right\|_{V}+\left\|v_{0}\right\|_{V}+\left\|F_{\ell}^{*}\right\|_{L^{\infty}\left(0, t ; V^{*}\right)}\right), \quad \forall t \in[0, T] .
$$


Next, we substitute $z=w_{t}(t)$ in $(23)$ to obtain

$$
\begin{gathered}
\left\|w_{t}(t)\right\|_{V}^{2}+\int_{-L}^{L} p(x, t) w_{x}(t) w_{t x}(t) d x-\int_{-L}^{L} \varphi^{\prime}\left(v_{0}\right)\left(v_{0}\right)_{x}(t) w(t) w_{t x}(t) d x \\
=\int_{-L}^{L} F\left(x, t, n_{0}, v_{0}\right) w_{t}(t) d x+\left\langle F_{\ell}^{*}(t), w_{t}(t)\right\rangle_{V^{*}, V}, \quad \text { a.e. } t \in(0, T) .
\end{gathered}
$$

Here, we use (A1)-(A5) again and obtain the following estimates:

$$
\begin{aligned}
& -\int_{-L}^{L} p(x, t)\left|w_{x}(t)\left\|w_{t x}(t) \mid d x \leq \delta\right\| w_{t}(t)\left\|_{V}^{2}+\frac{c_{1}^{2}}{4 \kappa^{2} \delta}\right\| w(t) \|_{V}^{2} ;\right. \\
& \text { - } \int_{-L}^{L}\left|\varphi^{\prime}\left(v_{0}\right)\left\|\left(v_{0}\right)_{x}\right\| w(t)\left\|w_{t x}(t) \mid d x \leq \delta\right\| w_{t}(t)\left\|_{V}^{2}+\frac{c_{3}^{2} L(\varphi)^{2}\left\|v_{0}\right\|_{V}^{2}\|w(t)\|_{V}^{2} ;}{4 \kappa^{2} \delta}\right\|\right. \\
& \text { - } \int_{-L}^{L}\left|F\left(x, t, n_{0}, v_{0}\right)\left\|w_{t}(t) \mid d x \leq \delta\right\| w_{t}(t) \|_{V}^{2}+\frac{c_{3}^{2}}{2 \delta}\left(\left\|n_{0}\right\|_{V}^{2}+\left\|v_{0}\right\|_{V}^{2}\right) .\right.
\end{aligned}
$$

By substituting the above estimates in (29) and taking $\delta=\frac{1}{8}$, we see that there exist constants $C_{5}>0$ and $C_{6} \geq 2$ such that

$$
\left\|w_{t}(t)\right\|_{V} \leq C_{5}\|w(t)\|_{V}+C_{6}\left(\left\|n_{0}\right\|_{V}+\left\|v_{0}\right\|_{V}+\left\|F_{\ell}^{*}(t)\right\|_{V^{*}}\right), \quad \text { a.e. } t \in(0, T)
$$

Finally, by putting

$$
h_{1}(t)=\left(1+C_{5}\right) \sqrt{\left(1+C_{4} t\right) e^{C_{3} t}}+C_{6}, \quad \forall t \geq 0,
$$

we see from (28) and (30) that (25) holds.

4. Local existence of solutions. The argument carried out in this section is essentially the same as in [6]. First of all, we show the following lemma.

Lemma 4.1. For any $\varepsilon>0$ there exists $T_{\varepsilon} \in(0, T]$ such that for any $t \in\left(0, T_{\varepsilon}\right]$

$$
\|\ell\|_{W^{1, \infty}(0, t ; V)} \leq \varepsilon\left(\left\|n_{0}\right\|_{V}+\left\|v_{0}\right\|_{V}\right) \quad \Longrightarrow \quad\left\|F_{\ell}^{*}\right\|_{L^{\infty}\left(0, t ; V^{\star}\right)} \leq\left\|n_{0}\right\|_{V} .
$$

Proof. Let $t \in[0, T]$ and $s \in[0, t]$. First, we see from (A3) that

$$
\begin{aligned}
& \left|\int_{-L}^{L}\left\{F\left(x, s, \ell(s), \eta_{\ell}(s)\right)-F\left(x, s, n_{0}, v_{0}\right)\right\} z d x\right| \\
\leq & c_{2}\left(\left\|\ell(s)-n_{0}\right\|_{H}+\left\|\eta_{\ell}(s)-v_{0}\right\|_{H}\right)\|z\|_{H} \\
\leq & c_{2}\left(\int_{0}^{s}\left\|\left(\frac{d \ell_{1}}{d \tau}\right)(\tau)\right\|_{H} d \tau+\nu \int_{0}^{s}\|\ell(\tau)\|_{H} d \tau\right)\|z\|_{H} \\
\leq & c_{2}(1+\nu) t\|\ell\|_{W^{1, \infty}(0, t ; V)}\|z\|_{V} .
\end{aligned}
$$


Second, we see from (A2) and (18) that

$$
\begin{aligned}
& \left|\int_{-L}^{L} \ell(s)\left(\varphi\left(\eta_{\ell}(s)\right)-\varphi\left(v_{0}\right)\right)_{x} z_{x} d x\right| \\
\leq & \int_{-L}^{L}\left|\ell(s)\left\|\varphi^{\prime}\left(\eta_{\ell}(s)\right)\left(\eta_{\ell}\right)_{x}(t)-\varphi^{\prime}\left(v_{0}\right)\left(v_{0}\right)_{x}\right\| z_{x}\right| d x \\
\leq & L(\varphi) \int_{-L}^{L}\left|\ell(s)\left\|\left(\eta_{\ell}\right)_{x}(s)-\left(v_{0}\right)_{x}\right\| z_{x}\right| d x+L\left(\varphi^{\prime}\right) \int_{-L}^{L}\left|\ell(s)\left\|\eta_{\ell}(s)-v_{0}\right\|\left(v_{0}\right)_{x} \| z_{x}\right| d x \\
\leq & \nu c_{3} L(\varphi)\|\ell(s)\|_{V} \int_{0}^{s} \int_{-L}^{L}\left|\ell_{x}(\tau) \| z_{x}\right| d x d \tau \\
& +\nu c_{3} L\left(\varphi^{\prime}\right)\|\ell(s)\|_{V} \int_{0}^{s} \int_{-L}^{L}\left|\ell(\tau)\left\|\left(v_{0}\right)_{x}\right\| z_{x}\right| d x d \tau \\
\leq & \frac{\nu c_{3}\left\{L(\varphi)+c_{3} L\left(\varphi^{\prime}\right)\left\|v_{0}\right\|_{V}\right\}}{\kappa}\|\ell(s)\|_{V}\left(\int_{0}^{s}\|\ell(\tau)\|_{V} d \tau\right)\|z\|_{V} \\
\leq & \frac{\nu c_{3}\left\{L(\varphi)+c_{3} L\left(\varphi^{\prime}\right)\left\|v_{0}\right\|_{V}\right\}}{\kappa} t\|\ell\|_{L^{\infty}(0, t ; V)}^{2}\|z\|_{V} \cdot
\end{aligned}
$$

Hence, we derive

$$
\left\|F_{\ell}^{*}\right\|_{L^{\infty}\left(0, t ; V^{\star}\right)} \leq C_{7}\|\ell\|_{W^{1, \infty}(0, t ; V)}\left(1+\|\ell\|_{W^{1, \infty}(0, t ; V)}\right) t, \quad \forall t \in[0, T],
$$

where

$$
C_{7}=c_{2}(1+\nu)+\frac{\nu c_{3}\left\{L(\varphi)+c_{3} L\left(\varphi^{\prime}\right)\left\|v_{0}\right\|_{V}\right\}}{\kappa} .
$$

Finally, we put

$$
T_{\varepsilon}=\min \left\{T, \frac{1}{C_{7} \varepsilon\left(\left\|n_{0}\right\|_{V}+\left\|v_{0}\right\|_{V}\right)\left\{1+\varepsilon\left(\left\|n_{0}\right\|_{V}+\left\|v_{0}\right\|_{V}\right\}\right.}\right\} .
$$

Then, it is easily seen from (31) that the inequality

$$
\left\|F_{\ell}^{*}\right\|_{L^{\infty}\left(0, t ; V^{\star}\right)} \leq\left\|n_{0}\right\|_{V}
$$

holds for any $t \in\left(0, T_{\varepsilon}\right]$ and $\ell$ satisfying $\|\ell\|_{W^{1, \infty}(0, t ; V)} \leq \varepsilon\left(\left\|n_{0}\right\|_{V}+\left\|v_{0}\right\|_{V}\right)$. Hence, this lemma is completely proved.

Next, for each $t \in(0, T]$ we consider a closed subset $X_{t}$ of $W^{1, \infty}(0, t ; V)$ given by

$$
X_{t}:=\left\{\ell \in W^{1, \infty}(0, t ; V) \mid \ell(0)=n_{0} \text { and }\|\ell\|_{W^{1, \infty}(0, t ; V)} \leq 2 h_{1}(T)\left(\left\|n_{0}\right\|_{V}+\left\|v_{0}\right\|_{V}\right)\right\},
$$

where $h_{1}$ is the same function as obtained in Proposition 3.1. It is clear that $X_{t} \neq \emptyset$ for any $t \in[0, T]$ since $n_{0} \in X_{t}$.

By applying Lemma 4.1, we see that the following property ( $\star$ ) is satisfied:

$$
\text { (*) } \quad\left(\begin{array}{c}
\text { there exists } T_{1} \in(0, T] \text { such that for any } t \in\left(0, T_{1}\right] \\
\left\|F_{\ell}^{*}\right\|_{L^{\infty}\left(0, t ; V^{*}\right)} \leq\left\|n_{0}\right\|_{V}, \quad \forall \ell \in X_{t} .
\end{array}\right.
$$


Moreover, it follows from Proposition 3.1 with $(\star)$ that for any $t \in\left(0, T_{1}\right]$

$$
\begin{aligned}
\left\|w_{\ell}\right\|_{W^{1, \infty}(0, t ; V)} & \leq h_{1}(t)\left(\left\|n_{0}\right\|_{V}+\left\|v_{0}\right\|_{V}+\left\|F_{\ell}^{*}\right\|_{L^{\infty}\left(0, t ; V^{*}\right)}\right) \\
& \leq 2 h_{1}(T)\left(\left\|n_{0}\right\|_{V}+\left\|v_{0}\right\|_{V}\right),
\end{aligned}
$$

where $w_{\ell}$ is a unique solution to $(\mathrm{AP})_{\ell}$ on $[0, t]$, which implies $w_{\ell} \in X_{t}$. That is, for each $t \in\left(0, T_{1}\right]$ we can define a solution operator $S_{t}$ from $X_{t}$ into itself by

$$
S_{t} \ell:=w_{\ell}, \quad \forall \ell \in X_{t} .
$$

Then, we derive the following lemma.

Lemma 4.2. There exists $T_{2} \in\left(0, T_{1}\right]$ such that for any $t \in\left(0, T_{2}\right)$ the operator $S_{t}$ is a contraction mapping on $X_{t}$.

Proof. Let $t \in\left(0, T_{1}\right], \ell_{i} \in X_{t}$ and $w_{i}=S_{t} \ell_{i}(i=1,2)$. Then, we see that $W=w_{1}-w_{2}$ satisfies the following equality:

$$
\begin{gathered}
\left(W_{s}(s), z\right)_{V}+\int_{-L}^{L} p(x, s) W_{x}(s) z_{x} d x-\int_{-L}^{L}\left(\varphi\left(v_{0}\right)\right)_{x} W(s) z_{x} d x \\
=\left\langle F_{\ell_{1}}^{*}(s)-F_{\ell_{2}}^{*}(s), z\right\rangle_{V^{*}, V}, \quad \forall z \in V, \text { a.e. } s \in(0, t) .
\end{gathered}
$$

In order to get some estimate of $F_{\ell_{1}}^{*}-F_{\ell_{2}}^{*}$, we carry out the following argument. First, by repeating the similar argument as in the first half of Lemma 4.1, we have:

$$
\begin{aligned}
& \left|\int_{-L}^{L}\left\{F\left(x, s, \ell_{1}(s), \eta_{\ell_{1}}(s)\right)-F\left(x, s, \ell_{2}(s), \eta_{\ell_{2}}(s)\right)\right\} z d x\right| \\
& \leq c_{2}(1+\nu) t\left\|\ell_{1}-\ell_{2}\right\|_{W^{1, \infty}(0, t ; V)}\|z\|_{V}, \quad \text { a.e. } s \in(0, t) .
\end{aligned}
$$

Second, we have:

$$
\begin{aligned}
& \left|\int_{-L}^{L} \ell_{1}(s)\left(\varphi\left(\eta_{\ell_{1}}(s)\right)-\varphi\left(v_{0}\right)\right)_{x} z_{x} d x-\int_{-L}^{L} \ell_{2}(s)\left(\varphi\left(\eta_{\ell_{2}}(s)\right)-\varphi\left(v_{0}\right)\right)_{x} z_{x} d x\right| \\
\leq & \int_{-L}^{L}\left|\varphi^{\prime}\left(\eta_{\ell_{1}}(s)\right)\right|\left|\left(\eta_{\ell_{1}}\right)_{x}(s)\right|\left|\ell_{1}(s)-\ell_{2}(s)\right|\left|z_{x}\right| d x \\
& +\int_{-L}^{L}\left|\ell_{2}(s)\left\|\left(\eta_{\ell_{1}}\right)_{x}(s)\right\| \varphi^{\prime}\left(\eta_{\ell_{1}}(s)\right)-\varphi^{\prime}\left(\eta_{\ell_{2}}(s)\right) \| z_{x}\right| d x \\
& +\int_{-L}^{L}\left|\ell_{2}(s)\left\|\varphi^{\prime}\left(\eta_{\ell_{2}}(s)\right)||\left(\eta_{\ell_{1}}\right)_{x}(s)-\left(\eta_{\ell_{2}}\right)_{x}(s)\right\| z_{x}\right| d x \\
& +\int_{-L}^{L}\left|\varphi^{\prime}\left(v_{0}\right)\left\|\left(v_{0}\right)_{x}\right\| \ell_{1}(s)-\ell_{2}(s) \| z_{x}\right| d x \\
=: & I_{1}(s)+I_{2}(s)+I_{3}(s)+I_{4}(s) .
\end{aligned}
$$

We estimate the integrals $I_{i}(1 \leq i \leq 4)$ below. By using (A2), (A5) and (18), we derive 
the following estimates:

- $I_{1}(s) \leq \nu c_{3} L(\varphi)\left\|\ell_{1}(s)-\ell_{2}(s)\right\|_{V} \int_{-L}^{L}\left|\int_{0}^{s}\left(\ell_{1}\right)_{x}(\tau) d \tau\right|\left|z_{x}\right| d x$

$$
\begin{aligned}
& \leq \frac{\nu c_{3} L(\varphi)\left\|\ell_{1}(s)-\ell_{2}(s)\right\|_{V}}{\kappa}\left(\int_{0}^{s}\left\|\ell_{1}(\tau)\right\|_{V} d \tau\right)\|z\|_{V} \\
& \leq \frac{\nu c_{3} L(\varphi)\left\|\ell_{1}\right\|_{L^{\infty}(0, t ; V)} t\left\|\ell_{1}-\ell_{2}\right\|_{W^{1, \infty}(0, t ; V)}\|z\|_{V}}{\kappa} \\
& \leq \frac{2 \nu c_{3} L(\varphi) h_{1}(T)\left(\left\|n_{0}\right\|_{V}+\left\|v_{0}\right\|_{V}\right)}{\kappa} t\left\|\ell_{1}-\ell_{2}\right\|_{W^{1, \infty}(0, t ; V)}\|z\|_{V} ;
\end{aligned}
$$

- $I_{2}(s) \leq c_{3} L\left(\varphi^{\prime}\right)\left\|\ell_{2}(s)\right\|_{V} \int_{-L}^{L}\left|\int_{0}^{s}\left\{\ell_{1}(\tau)-\ell_{2}(\tau)\right\} d \tau\right|\left|\left(\eta_{\ell_{1}}\right)_{x}(s)\right|\left|z_{x}\right| d x$

$$
\begin{aligned}
\leq & c_{3} L\left(\varphi^{\prime}\right)\left\|\ell_{2}(s)\right\|_{V} \int_{0}^{s} \int_{-L}^{L}\left|\ell_{1}(\tau)-\ell_{2}(\tau)\left\|\left(v_{0}\right)_{x}\right\| z_{x}\right| d x \\
& +\nu c_{3} L\left(\varphi^{\prime}\right)\left\|\ell_{2}(s)\right\|_{V} \int_{-L}^{L}\left(\int_{0}^{s}\left|\ell_{1}(\tau)-\ell_{2}(\tau)\right| d \tau\right)\left(\int_{0}^{s}\left|\left(\ell_{1}\right)_{x}(\tau)\right|\right)\left|z_{x}\right| d x \\
\leq & \frac{c_{3}^{2} L\left(\varphi^{\prime}\right)\left\|\ell_{2}(s)\right\|_{V}\left\|v_{0}\right\|_{V}}{\kappa}\left(\int_{0}^{s}\left\|\ell_{1}(\tau)-\ell_{2}(\tau)\right\|_{V} d \tau\right)\|z\|_{V} \\
& +\frac{\nu c_{3}^{2} L\left(\varphi^{\prime}\right)\left\|\ell_{2}(s)\right\|_{V}}{\kappa}\left(\int_{0}^{s}\left\|\ell_{1}(\tau)-\ell_{2}(\tau)\right\|_{V} d \tau\right)\left(\int_{0}^{s}\left\|\ell_{1}(\tau)\right\|_{V} d \tau\right)\|z\|_{V} \\
\leq & \frac{c_{3}^{2} L\left(\varphi^{\prime}\right)\left\|\ell_{2}\right\|_{L^{\infty}(0, t ; V)}\left(\left\|v_{0}\right\|_{V}+\nu\left\|_{1}\right\|_{L^{\infty}(0, t ; V)}\right)}{\kappa} \\
& \times t(1+t)\left\|\ell_{1}-\ell_{2}\right\|_{L^{\infty}(0, t ; V)}\|z\|_{V} \\
\leq & \frac{2 c_{3}^{2} L\left(\varphi^{\prime}\right) h_{1}(T)\left\{1+2 \nu h_{1}(T)\right\}\left(\left\|n_{0}\right\|_{V}+\left\|v_{0}\right\|_{V}\right)^{2}}{\kappa} \\
& \times t(1+t)\left\|\ell_{1}-\ell_{2}\right\|_{L^{\infty}(0, t ; V)}\|z\|_{V} ;
\end{aligned}
$$

- $I_{3}(s) \leq \nu c_{3} L(\varphi)\left\|\ell_{2}(s)\right\|_{V} \int_{-L}^{L}\left|\int_{0}^{s}\left\{\left(\ell_{1}\right)_{x}(\tau)-\left(\ell_{2}\right)_{x}(\tau)\right\} d \tau\right|\left|z_{x}\right| d x$

$$
\begin{aligned}
& \leq \frac{\nu c_{3} L(\varphi)\left\|\ell_{2}(s)\right\|_{V}}{\kappa}\left(\int_{0}^{s}\left\|\ell_{1}(\tau)-\ell_{2}(\tau)\right\|_{V} d \tau\right)\|z\|_{V} \\
& \leq \frac{\nu c_{3} L(\varphi)\left\|\ell_{2}\right\|_{L^{\infty}(0, t ; V)}}{\kappa} t\left\|\ell_{1}-\ell_{2}\right\|_{L^{\infty}(0, t ; V)}\|z\|_{V} \\
& \leq \frac{2 \nu c_{3} L(\varphi) h_{1}(T)\left(\left\|n_{0}\right\|_{V}+\left\|v_{0}\right\|_{V}\right)}{\kappa} t\left\|\ell_{1}-\ell_{2}\right\|_{L^{\infty}(0, t ; V)}\|z\|_{V}
\end{aligned}
$$




$$
\begin{aligned}
I_{4}(s) & \leq c_{3} L(\varphi)\left\|\ell_{1}(s)-\ell_{2}(s)\right\|_{V} \int_{-L}^{L}\left|\left(v_{0}\right)_{x} \| z_{x}\right| d x \\
& \leq \frac{c_{3} L(\varphi)}{\kappa}\left(\int_{0}^{s}\left\|\left(\frac{d}{d s}\left(\ell_{1}-\ell_{2}\right)\right)(s)\right\|_{V} d s\right)\left\|v_{0}\right\|_{V}\|z\|_{V} \\
& \leq \frac{c_{3} L(\varphi)\left\|v_{0}\right\|_{V}}{\kappa} t\left\|\frac{d}{d s}\left(\ell_{1}-\ell_{2}\right)\right\|_{L^{\infty}(0, t ; V)}\|z\|_{V} .
\end{aligned}
$$

We see from the above estimates that there exists a constant $C_{8}(T)>0$ such that

$$
\begin{aligned}
& \mid \int_{-L}^{L} \ell_{1}(s)\left(\varphi\left(\eta_{\ell_{1}}(s)\right)-\right.\left.\varphi\left(v_{0}\right)\right)_{x} z_{x} d x-\int_{-L}^{L} \ell_{2}(s)\left(\varphi\left(\eta_{\ell_{2}}(s)\right)-\varphi\left(v_{0}\right)\right)_{x} z_{x} d x \mid \\
& \leq C_{8}(T) t(1+t)\left\|\ell_{1}-\ell_{2}\right\|_{W^{1, \infty}(0, t ; V)}\|z\|_{V} \quad \text { a.e. } s \in(0, t) .
\end{aligned}
$$

Hence, it follows from (33) and (34) that there exists a constnat $C_{9}(T)>0$ such that

$$
\left\|F_{\ell_{1}}^{*}-F_{\ell_{2}}^{*}\right\|_{L^{\infty}\left(0, t ; V^{*}\right)} \leq C_{9}(T) t(1+t)\left\|\ell_{1}-\ell_{2}\right\|_{W^{1, \infty}(0, t ; V)} .
$$

Next, we substitute $z=W(s)$ in (32). By repeating the similar argument as in the proof of Proposition 3.1 and using (35), we see that there exists a constant $C_{10}>0$ such that

$$
\|W\|_{L^{\infty}(0, t ; V)} \leq C_{9}(T) t^{\frac{3}{2}}(1+t) e^{C_{10} t}\left\|\ell_{1}-\ell_{2}\right\|_{W^{1, \infty}(0, t ; V)} .
$$

Moreover, by substituting $z=W_{s}(s)$ in (32) and repeating the similar argument as in the latter half of the proof of Proposition 3.1 again, we see that there exists a constant $C_{11}>0$ such that

$$
\left\|W_{s}\right\|_{L^{\infty}(0, t ; V)} \leq C_{11}\left(\|W\|_{L^{\infty}(0, t ; V)}+\left\|F_{\ell_{1}}^{*}-F_{\ell_{2}}^{*}\right\|_{L^{\infty}(0, t ; V)}\right) .
$$

Hence, we see from (35)-(37) that

$$
\|W\|_{W^{1, \infty}(0, t ; V)} \leq\left(1+C_{11}\right) C_{9}(T) h_{2}(t)\left\|\ell_{1}-\ell_{2}\right\|_{W^{1, \infty}(0, t ; V)} .
$$

where $h_{2}$ is a function given by

$$
h_{2}(t)=\left(\sqrt{t} e^{C_{10} t}+1\right) t(1+t), \quad \forall t \in[0,+\infty) .
$$

Since the function $h_{2}$ is a strictly increasing and continuous function satisfying $h_{2}(0)=0$ and $\lim _{t \rightarrow+\infty} h_{2}(t)=+\infty$, we see that there exists a unique $\tilde{T}_{2} \in(0,+\infty)$ such that

$$
h_{2}\left(\tilde{T}_{2}\right)=\frac{1}{\left(1+C_{2}\right) A_{2}(T)} .
$$

Finally, we put $T_{2}=\min \left\{T_{1}, \tilde{T}_{2}\right\}$. Then, we see from (38) that for any $t \in\left(0, T_{2}\right)$ there exists a constant $h_{t} \in(0,1)$ such that

$$
\left\|S_{t} \ell_{1}-S_{t} \ell_{2}\right\|_{W^{1, \infty}(0, t ; V)} \leq h_{t}\left\|\ell_{1}-\ell_{2}\right\|_{W^{1, \infty}(0, t ; V)}, \quad \forall \ell_{1}, \ell_{2} \in X_{t},
$$

which implies that $S_{t}$ is a contraction mapping on $X_{t}$.

Now, we give the main theorem of this section.

THEOREM 4.1. Let $T_{2}$ be the same number as obtained in Lemma 4.2. Then, for each $t \in\left(0, T_{2}\right)(P)$ has at least one solution $[n, v]$ on $[0, t]$. 
Proof. By applying the Banach fixed point theorem to $S_{t}$, we see that $S_{t}$ has a unique fixed point $n \in X_{t}$, i.e., $S_{t} n=n$. And we put

$$
v(x, s)=v_{0}(x)+\nu \int_{0}^{s} n(x, \tau) d \tau, \quad \forall(x, s) \in[-L, L] \times[0, t] .
$$

Then, it is easily checked that $[n, v]$ is a solution to $(\mathrm{P})$ on $[0, t]$.

5. Iteration scheme. In this section, we give one of the methods to approximate the solutions to $(\mathrm{P})$. Actually, this is a direct consequence of Sections 2 and 4 . For this, we fix $T_{0} \in\left(0, T_{2}\right)$ throughout this section, where $T_{2}$ is the same number as obtained in Lemma 4.2 .

Now, we define a sequence $\left\{\left[n^{k}, v^{k}\right]\right\}_{k \in\{0\} \cup \mathbf{N}}$ by the following way:

$$
\left\{\begin{array}{l}
n^{0}(x, t)=n_{0}(x), \\
v^{0}(x, t)=v_{0}(x)+\int_{0}^{t} n^{0}(x, s) d s,
\end{array} \quad \forall(x, t) \in[-L, L] \times[0, T]\right.
$$

and for any $k \in \mathbf{N}$

$$
\left\{\begin{array}{l}
n^{k+1}(x, t)=\left[S_{T_{0}} n^{k}\right](x, t), \\
v^{k+1}(x, t)=v_{0}(x)+\int_{0}^{t} n^{k+1}(x, s) d s,
\end{array} \quad \forall(x, t) \in[-L, L] \times[0, T] .\right.
$$

Since $n_{0} \in X_{T_{0}}$ and $T_{0} \in\left(0, T_{2}\right)$, it is clear that $n^{k} \in X_{T_{0}}$ for any $k \in\{0\} \cup \mathbf{N}$. Moreover, we see that the following theorem holds.

THEOREM 5.1. The sequence $\left[n^{k}, v^{k}\right]$ converges to $[n, v]$ strongly in $W^{1, \infty}\left(0, T_{0}, V\right) \times$ $W^{2, \infty}\left(0, T_{0} ; V\right)$ as $k \rightarrow \infty$, where $[n, v]$ is a unique solution to $(P)$ on $\left[0, T_{0}\right]$.

Proof. From Lemma 4.2, we see that $n^{k}$ converges to $n$, which is a unique fixed point of $S_{T_{0}}$, strongly in $W^{1, \infty}\left(0, T_{0} ; V\right)$ as $k \rightarrow \infty$. Hence, it is also easily obtained from the definition of $v^{k}$ that it converges to $v$ strongly in $W^{2, \infty}\left(0, T_{0} ; V\right)$ as $k \rightarrow \infty$. At last, we see from Theorems 2.1 and 4.1 that $[u, v]$ is a unique solution to $(\mathrm{P})$ on $\left[0, T_{0}\right]$.

Acknowldgements. This research was accomplished while A. Ito stayed at ICM, Warsaw University, as the long-term researcher overseas of Kinki University.

\section{References}

[1] M. A. J. Chaplain and A. R. A. Anderson, Mathematical modelling of tissue invasion, in: L. Preziosi (eds.), Cancer Modelling and Simulation, Chapman \& Hall/CRC, Boca Raton, 2003, 269-297.

[2] L. Corrias, B. Perthame and H. Zaag, A chemotaxis model motivated by angiogenesis, C. R. Acad. Sci. Paris Ser. I 336 (2003), 141-146.

[3] L. Corrias, B. Perthame and H. Zaag, Global solutions of some chemotaxis and angiogenesis systems in high space dimensions, Milan J. Math. 72 (2004), 1-28.

[4] M. A. Fontelos, A. Friedman and B. Hu, Mathematical analysis of a model for the initiation of angiogenesis, SIAM J. Math. Anal. 33 (2002), 1330-1355. 
[5] A. Friedman and J. I. Tello, Stability of solutions of chemotaxix equations in reinforced random walks, J. Math. Anal. Appl. 272 (2002), 138-163.

[6] R. Takahashi and T. Suzuki, Global in time solution to a class of tumor growth systems, preprint. 